\title{
Fatigue, Countermeasures, and Performance Enhancement in Resident Physicians
}

$\mathrm{T}$ The effects of fatigue, night shift assignments, and sleep deprivation on human performance and the quality and safety of medical care are subjects of enormous interest within and beyond the medical community. Much of the focus has been on the duty hours of resident physicians, the effects of fatigue on their clinical performance, and the impact of duty assignments on resident learning and well-being.

Duty hour restrictions for resident physicians in training programs accredited by the Accreditation Council for Graduate Medical Education (ACGME) were implemented on July 1, 2003, in response to concerns that fatigue contributes to medical errors. ${ }^{1}$ Since then, a number of studies have suggested that reducing resident work hours and modifying their duty shifts may lead to fewer attention failures, fewer medical errors, and greater resident well-being. ${ }^{2,3}$ However, even in the presence of these desirable metrics, few outcome data have documented that resident duty hour restrictions have indeed improved the overall quality of patient care. ${ }^{4,5}$ Nonetheless, the Institute of Medicine (IOM) recently published an extensive report addressing this issue in which it recommends further restriction of resident physician duty hours. ${ }^{6}$ The most controversial IOM recommendation would allow residents to admit patients for only 16 hours and require a 5-hour protected sleep period each night. The ACGME is currently studying the IOM recommendations and other available data to further assess resident duty hour requirements. ${ }^{4,5}$

Ideally, resident assignments and duty hour restrictions would be based on results of well-conducted trials that specifically address the impact of resident fatigue, night shift assignments, shift length, and time between shifts on

Address correspondence to Steven H. Rose, MD, Department of Anesthesiology, Mayo Clinic, 200 First St SW, Rochester, MN 55905 (rose.steven @mayo.edu).

(C) 2009 Mayo Foundation for Medical Education and Research the quality and safety of patient care, on resident learning, and on resident safety (eg, needle sticks and motor vehicle crashes). ${ }^{7-9}$ Duty hour requirements should also promote residents' physical and mental health. Unfortunately, the scale and scope of these issues and individual variability in the need for sleep make it unlikely that sufficient data will soon be available to guide these decisions.

In this issue of Mayo Clinic Proceedings, Czeisler et $\mathrm{al}^{10}$ report the results of a well-designed study that adds another piece to this complex puzzle. They conducted a large, multicenter, industry-funded, randomized clinical trial that compares the effects of armodafinil (Nuvigil; Cephalon Inc, Frazer, PA) to placebo in treatment of excessive sleepiness associated with shift work disorder (SWD) (a circadian rhythm

See also page 958 sleep disorder). The authors report significant improvements in sleep latency and performance on standardized memory and attention tests along with reduced self-reported sleepiness during night shifts and the commute home in the treatment group

The ACGME and IOM have attempted to address the impact of resident physician fatigue through work schedule restrictions. Czeisler et al tested an alternative approach (in nonphysician study participants) by using a drug to treat the symptoms of SWD. Extending the use of drug therapy to include resident physicians with no identified sleep disorder to improve concentration and learning, improve wakefulness, enhance performance, and promote highquality patient care (especially at night) raises a variety of concerns.

The use of drugs to sustain and improve performance during sleep deprivation is not a novel concept. For example, pilot fatigue is a major concern in aviation, and fatigue continues to be cited as a cause of near-miss events, errors, and crashes despite strict pilot flight hour regulation. Modafinil, a racemic mixture of armodafinil with a shorter duration of action than the R-enantiomer armodafinil, is 
approved by the US Air Force for use by bomber pilots conducting missions lasting more than 12 hours. ${ }^{11}$ However, the US Air Force places substantial restrictions on its use, including limiting it to 2-pilot missions (with only 1 of the pilots taking the drug), documentation of the exhaustion of nonpharmacological countermeasures, pre-use counseling, informed consent, prerequisite testing of the drug in nonflight situations, use for no longer than 3 months, and postmission follow-up and documentation. Even with such safeguards, there are important ethical considerations in recommending use of drugs for cognitive enhancement among civilian health care professionals, including individual choice (autonomy), safety (for the person taking the drug and his/her patients), and necessity. ${ }^{12}$ Issues such as cross-cultural considerations also need to be considered.

Important differences exist between the population in the study by Czeisler et al and typical resident physicians. Study participants were between 18 and 65 years of age, worked more than 5 night shifts per month of less than 12 hours' duration, and worked at least 3 consecutive night shifts. Only individuals who met the diagnostic criteria for SWD of moderate or greater severity were eligible to participate in the study. In comparison, most resident physicians are young adults, often work shifts more than 12 hours, and are not permanently assigned to a night shift schedule so they rarely work night shifts for 3 or more consecutive days. The prevalence of SWD of moderate or greater severity in resident physicians is unknown, and it is unclear whether residents could be routinely tested for this disorder or have the results of such testing made available to their program director without violating rights to privacy. Still, the results of the current study have potential to influence the resident duty hour debate.

The ACGME has traditionally regarded night assignments as an important educational activity for resident physicians, providing them with opportunities for continuity of experience in managing disease processes as they evolve during a 24-hour period. This experience could be compromised if stricter requirements regarding duty hours are enacted, resulting in suboptimal training. The data from the current study suggest that pharmacological agents may be used safely to counteract the effects of fatigue from prolonged work shifts and/or night assignments to preserve and/or enhance the continuity experience without sacrificing the quality of patient care.

However, even if thought to be safe and effective, armodafinil and related drugs may have unintended effects, including some yet to be discovered. As an example, individuals treated with modafinil during sleep deprivation may be unable to recognize when their cognitive performance is impaired. ${ }^{13,14}$ One perceived benefit of long-duty assignments during training is development of the ability to recognize the effects of fatigue on performance and how they can be managed while being supervised. What would be the consequences of using drug therapy to combat the effects of fatigue or restricting duty hours during training when residents must work without duty hour restrictions after graduation? Would reliance on an approved performance-enhancing drug during supervised residency training make it more likely that graduates would migrate to using unsupervised (and possibly illegal) stimulants after graduation? Few data are available regarding the effect of performance-enhancing drugs on the ability to learn new information and develop new skills. Furthermore, even if a perfect drug is identified, ethical issues may prevent mandating or even recommending its use. What if, for personal, religious, or cultural reasons, residents refuse to take such drugs, even if benefit to patients is documented? Whether use of these or other performance-enhancing drugs by physicians, including residents, should be allowed even by those with a specific diagnosis such as SWD may be the most relevant and important issue.

A fundamental question to be addressed is whether drugs to counteract sleep deprivation (with or without a diagnosed sleep disorder) are necessary in the clinical environment when systems of relief should make restorative sleep possible. Even if adequate graduate medical education can be delivered with further duty hour restrictions, this approach raises concerns about the provision of patient care while residents are resting. If such care falls to staff physicians, what are the implications for their performance as clinicians and educators? Coverage by nonphysician professionals is a frequently suggested alternative but incurs substantial costs, and professional availability is limited. Resident physicians do not always use their personal time already allotted for only study and sleep. "Moonlighting" or other diversions during off-duty hours could violate the spirit and effectiveness of duty hour restrictions during training.

Can performance-enhancing drugs safely be used to support the long hours historically worked by residents? If benefits such as those described by Czeisler et a ${ }^{10}$ could be obtained without risk or adverse effects, it may be possible, but whether the public and medical community will accept this brave new world of enhancing the physical and cognitive performance of physicians remains to be seen.

Steven H. Rose, MD Timothy B. Curry, MD, PhD

Department of Anesthesiology Mayo Clinic Rochester, MN 
1. Common Program Requirements: Resident Duty Hours in the learning and work environment. ACGME. Effective July 1, 2007. http://www.acgme.org /acWebsite/dutyHours/dh_ComProgrRequirmentsDutyHours0707.pdf. Accessed September 18, 2009.

2. Landrigan CP, Rothschild JM, Cronin JW, et al. Effect of reducing interns' work hours on serious medical errors in intensive care units. $N$ Engl J Med. 2004;351(18):1838-1848.

3. Lockley SW, Cronin JW, Evans EE, et al; Harvard Work Hours, Health and Safety Group. Effect of reducing interns' weekly work hours on sleep and attentional failures. N Engl J Med. 2004;351(18):1829-1837.

4. Volpp KG, Rosen AK, Rosenbaum PR, et al. Mortality among patients in VA hospitals in the first 2 years following ACGME resident duty hour reform. JAMA. 2007;298(9):984-992.

5. Volpp KG, Rosen AK, Rosenbaum PR, et al. Mortality among hospitalized Medicare beneficiaries in the first 2 years following ACGME resident duty hour reform. JAMA. 2007;298(9):975-983.

6. Institute of Medicine. Resident Duty Hours: Enhancing Sleep, Supervision, and Safety. Released December 15, 2008. http://www.iom.edu /Reports/2008/Resident-Duty-Hours-Enhancing-Sleep-Supervision-and -Safety.aspx. Accessed October 15, 2009.

7. Arnedt JT, Owens J, Crouch M, Stahl J, Carskadon MA. Neurobehavioral performance of residents after heavy night call vs after alcohol ingestion. JAMA. 2005;294(9):1025-1033.
8. Ayas NT, Barger LK, Cade BE, et al. Extended work duration and the risk of self-reported percutaneous injuries in interns. JAMA. 2006; 296(9):1055-1062

9. Barger LK, Cade BE, Ayas NT, et al. Extended work shifts and the risk of motor vehicle crashes among interns. N Engl J Med. 2005;352(2):125134 .

10. Czeisler CA, Walsh JK, Wesnes KA, Arora S, Roth T. Armodafinil for treatment of excessive sleepiness associated with shift work disorder: a randomized controlled study. Mayo Clin Proc. 2009;84(11):958-972.

11. Taylor GP Jr, Keys RE. Modafinil and management of aircrew fatigue. Washington, DC: Department of the Air Force: December 2, 2003 http://www.hep.afrl.af.mil/HEPF/Policy/modafinil.pdf. Accessed September 18, 2009.

12. Russo MB. Recommendations for the ethical use of pharmacologic fatigue countermeasures in the U.S. military. Aviat Space Environ Med. 2007;78(5) (suppl):B119-B127.

13. Baranski JV, Pigeau RA. Self-monitoring cognitive performance during sleep deprivation: effects of modafinil, d-amphetamine and placebo. J Sleep Res. 1997;6(2):84-91

14. Gurtman CG, Broadbear JH, Redman JR. Effects of modafinil on simulator driving and self-assessment of driving following sleep deprivation. Hum Psychopharmacol. 2008;23(8):681-692.

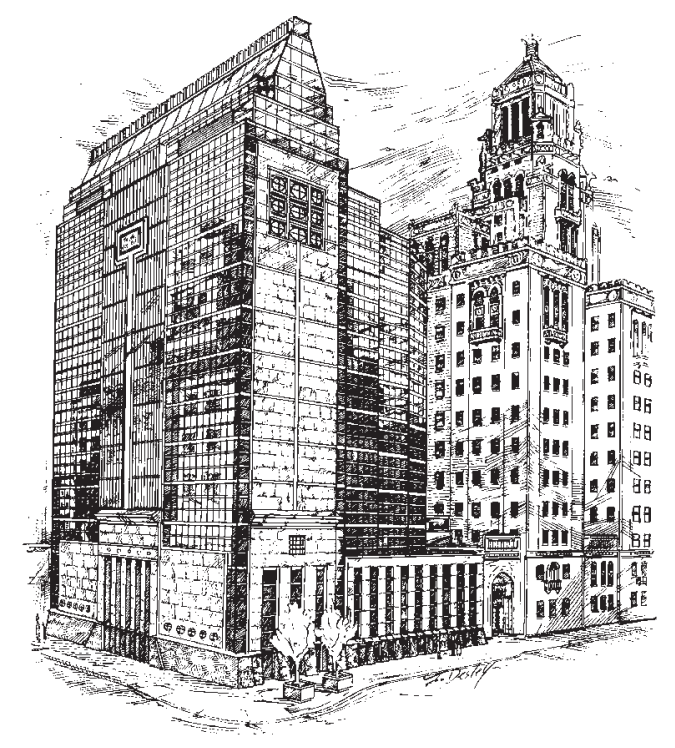

